\title{
Direct numerical simulation of infusion and flow-front tracking in materials with heterogeneous permeability using a pressure mapping sensor
}

Journal of Composite Materials $0(0) 1-15$

\author{
Arthur Levy' ${ }^{\oplus}$ and James Kratz ${ }^{2}$
}

\begin{abstract}
This paper explores the use of thin film piezoresistive pressure mapping sensors as a means to improve resin transfer moulding processes. The pressure mapping sensor was located between the preform and mould, giving information regarding the permeability map prior to infusion. The permeability map is used as an input to a direct numerical simulation of the infusion step of a highly variable reclaimed carbon fibre preform. The pressure sensor was also used to track the flow front position in-situ, due to a change in load sharing between the preform and liquid during the infusion experiment. Flow front tracking with the pressure mapping sensor was validated against conventional camera images taken through a transparent mould. The direct numerical simulation was able to account for local permeability variation in the preform, providing improved flow-front prediction than homogeneous permeability only, and could be part of a wider strategy to improve resin transfer moulding process robustness.
\end{abstract}

\section{Keywords}

Permeability, process monitoring, process simulation, liquid composite moulding

\section{Introduction}

Reclaimed carbon fibre materials are being introduced to market as composite waste recycling gathers momentum towards sustainable production. Challenges removing the polymer matrix from end-of-life parts has focused current recycling efforts towards ply cutter production waste recycling. Variability in the consolidation response and mechanical properties has been observed in reclaimed products created from dry fibre waste that is shredded, carded, and then sewn together, ${ }^{1}$ as visible in Figure 1. Addressing material variability in processing is required to ensure that landfill-diverted materials are successfully converted into recycled composite parts in manufacture.

Resin transfer moulding (RTM) technologies offer a flexible solution to create composite parts from a wide variety of reinforcement and matrix materials, and is particularly well suited to reclaimed fibre preforms. The manufacturing process has three main steps that will determine the quality of the finished composite part. First, intricate fibre preforms are created from linear layers of reinforcement plies or interlaced fibre networks. Second, the preform is placed into a mould tool where a liquid polymer is injected along a gate, saturating the fibre structure. Finally, the polymer is cured to form a high molecular weight glassy solid before the composite part is ejected from the mould.

Successful manufacture of composite parts by RTM requires a clear understanding of the material and process parameters that will govern polymer flow during filling. Darcy's Law is often used to describe the ease of fluid flow through a porous composite medium. ${ }^{2}$ For simple parts, back of the envelope flow front estimates using straight lines or elliptical curves for simple shapes can position the injection gate(s) such that air entrapment does not occur before the fluid reaches the vent(s). ${ }^{3}$ These defects are

\footnotetext{
'Laboratoire de Thermique et Energie de Nantes (LTEN), Nantes Université, France

${ }^{2}$ The Bristol Composites Institute (ACCIS), University of Bristol, UK

Corresponding author:

Arthur Levy, Laboratoire de Thermique et Energie de Nantes, Nantes Université, 44000 Nantes Cedex, France.

Email: arthur.levy@univ-nantes.fr
} 


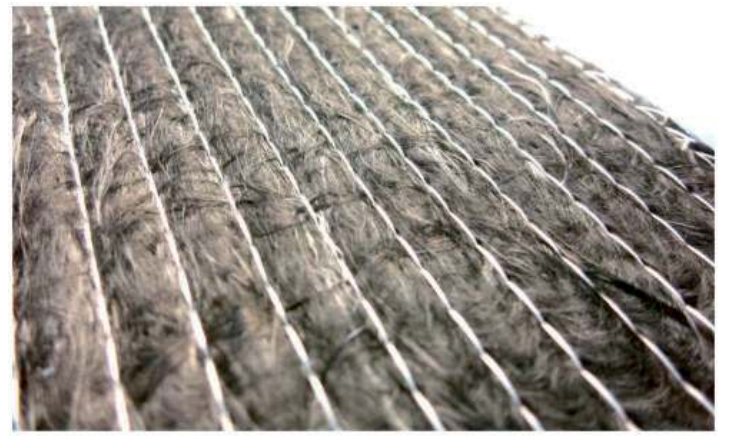

Figure I. Reclaimed carbon fibre dry material used in this study. Short randomly poorly oriented fibres are stitched together to form a mat.

typically referred to as dry spots. For more complex preforms and geometries, flow modelling software has been developed to simulate filling. Advanced software (such as PAM-RTM, LIMS, or Moldex3D) can handle process anomalies, such as race-tracking if the preform does not fit the mould properly, but an estimate for permeability in the race-tracking region is required by the simulation to predict whether any dry spots will arise during infusion. ${ }^{4-7}$

Variability is often present in composite preforms, and may result in entrapment of air during filling that leads to dry spots in the final part. Previous studies have addressed material variability in discontinuous mats. For example, Caglar et al. ${ }^{7}$ have directly identified the permeability map using inverse methods, and Endruweit et al. $^{8}$ have investigated the geometrical morphology of the randomness of fibre bundles to assess their importance on permeability variation. A more pragmatic approach to material variability was investigated by Walbran et al., ${ }^{9}$ where permeability irregularities was associated to pressure variability between a mould and preform. An overarching theme in permeability variation is the role of fibre volume fraction, which leads to flow front deviations from the expected filling behaviour, but can be addressed using numerical simulation, as shown in Endruweit et al. ${ }^{10}$

Variations in the fibre volume fraction will also influence the local compaction behaviour. Toll and Manson ${ }^{11}$ derived an elastic transverse behaviour that can be applied to dry random mats during consolidation, such as mould closing in RTM. The fibrebed reaction pressure $P_{f b}$ during a compression experiment, can be described by

$$
P_{f b}=E f^{4} V_{f}^{A}
$$

where $E$ is the fibre Young modulus, $f$ is the orientation function that ranges from 0 for unidirectional materials and up to 0.64 for random planar mats, $V_{f}$ is the fibre volume fraction, and $A$ is the power index that ranges from 3 for $3 \mathrm{D}$ random orientation and up to 5 for planar random orientation.

As $P_{f b}$ is increased, the fibre volume fraction of the preform also increases, reducing the mobility of resin flow. ${ }^{2}$ The change in permeability, $\boldsymbol{K}$, with respect to compaction has been studied by several authors. ${ }^{12-14}$ Based on classical theory of flow through porous media, the Kozeny-Carman equation can describe the permeability tensor $\boldsymbol{K}$ as a function of the fibre volume fraction $V_{f}$ as

$$
\boldsymbol{K}=\boldsymbol{C} \frac{\left(1-V_{f}\right)^{3}}{V_{f}^{2}}
$$

where $C$ is a constant of the fibre network. ${ }^{12-14}$ The Kozeny-Carman relationship may not be well suited to the case of highly-aligned unidirectional fibrebeds, especially for the transverse permeability components. ${ }^{15}$ However, Gebart ${ }^{16}$ derived a relationship for unidirectional materials with high fibre volume fraction. In the case of reclaimed materials with random distribution of fibres and a low fibre volume content investigated in this paper, the classical Kozeny-Carman equation is suitable.

Experimental characterisation methods for the permeability tensor remains an ongoing area of research in the composites community. ${ }^{17} \mathrm{~A}$ standard test method has yet to be formalised; however, all methods require measuring a pressure drop along the flow front and the flow front arrival time. ${ }^{18}$ The position of the flow front can be found using transparent moulds and a camera, point pressure sensors, a variety of mechanical (ultrasonic) and optical (fibres), or electromagnetic (dielectric or direct current) sensors. ${ }^{19}$ Mechanical pressure sensors can provide information about resin arrival and pressure drop along the flow front, however, their size limits the spacing between sensors. Optimisation methods using only three ${ }^{7,20}$ or four ${ }^{21}$ pressure sensors have successfully estimated the flow front in a complex filling scenario.

Previous studies have combined modelling techniques that take into account material defects or race tracking, with sensing methods that monitor the process online. Sozer et al., ${ }^{21}$ Nielsen and Pitchumani ${ }^{22}$ and Hsiao and Advani $^{23}$ have all proposed closed-loop methodologies to actively modify process parameters to prevent dry-spot defect and ensure proper filling. Nonetheless, it requires an in-situ tracking of the flow front inside the mould. Use of transparent mould ${ }^{4,5,22}$ is a solution that cannot be scaled up to industrial processes, whereas dielectric ${ }^{21,23}$ or pressure ${ }^{6}$ sensors are usually point measurements only giving sparse processing information. Electric sensors do not provide pressure data at the measurement location, ${ }^{24}$ whereas thin film pressure mapping sensors provide highdensity pressure measurements with minimal intrusion to the preform. ${ }^{9,25,26}$ 
This paper builds on and combines the scientific literature covering process modelling, material variability, and in-situ measurement to investigate flow in RTM of reclaimed fibre composites. The new contribution of this work is using a commercial piezoresistive thin film pressure mapping sensor to initialise a direct numerical simulation of the infusion step in the RTM process by correlating the fibrebed pressure to the permeability field. Once placed inside a closed mould, the pressure sensor provides high-density in-situ quantitative pressure data for individual preform. The pressure mapping sensor offered the added bonus of tracking the flow front during the injection phase, to validate the direct numerical simulation. These techniques are part of a wider aim to deliver closed-loop control of RTM processes to ensure proper filling of every part.

\section{Experimental methods}

\section{Materials}

Commercially available continuous unidirectional and reclaimed carbon fibre materials were used in this study. Type 62 RECATEX non-woven complex with an areal density of $200 \mathrm{~g} / \mathrm{m}^{2}$ from SGL Automotive Carbon Fibers (ACF) was used as the reclaimed product, and is shown in Figure 1. A continuous fibre counterpart, also from SGL ACF, SIGRATEX C U320-0/ ST was supplied as a unidirectional non-crimp fabric (NCF) with an areal density of $320 \mathrm{~g} / \mathrm{m}^{2}$.

Fibrebed compaction curve. The compaction behaviour of the reclaimed carbon fibre mat was characterised using a parallel plate compression setup in a universal testing machine. Three circular samples consisting of five layers measuring $135 \mathrm{~cm}$ were compressed following the procedure suggested by Kelly et al. ${ }^{27}$ Nine successive ramps were performed with the initial 6-mm gap reducing to $2 \mathrm{~mm}$ at $1 \mathrm{~mm} / \mathrm{min}$. Each gap was held constant for $5 \mathrm{~min}$ to allow the material to fully $\operatorname{relax}^{27}$ at pressures ranging between 0 and $800 \mathrm{kPa}$. The sample thickness was measured using the average of two laser displacement sensors and the corresponding sample fibre volume fraction was calculated as follows

$$
V_{f}=\frac{m}{\rho A h}
$$

where $m$ is the preform mass, $\rho$ is the fibre density, $A$ is the surface area, and $h$ is the sample height. Figure 2 shows the fibrebed material compaction reaction pressure $P_{f b}$ as a function of the volume fraction $V_{f}$.

Poor fibre alignment in the reclaimed material limits the fibre volume fraction to a maximum of $25 \%$ for most manufacturing processes. The experimental curves can be described by the semi-empirical compaction model proposed by Toll and Manson ${ }^{11}$ (equation 1). The fitting constants are summarised in Table 1. The obtained values are consistent with the range expected from Toll and Manson ${ }^{11}$ : the reclaimed material being poorly oriented the values were found between that of a unidirectional and a perfectly random mat.

Lubrication effects. The compaction behaviour of fibrous reinforcement is influenced by the friction between fibres. During an injection process, the

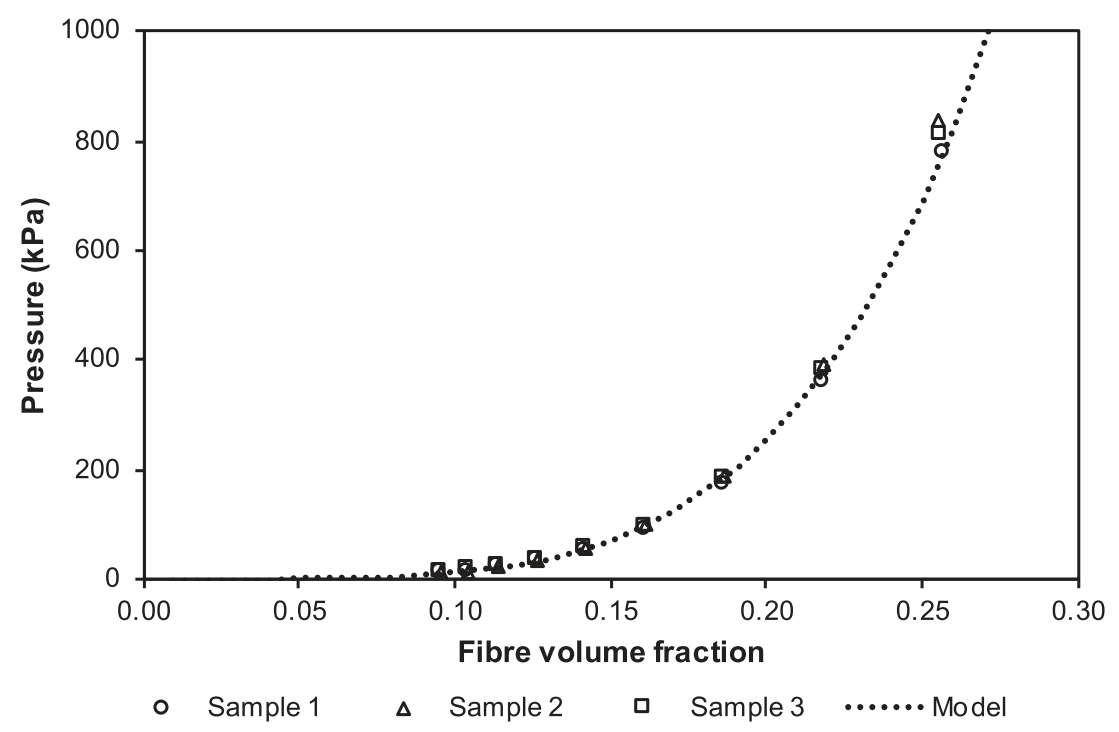

Figure 2. Fibrebed compaction curve for the reclaimed material. The model is given by equation (I). Because of the poor orientation, the fibre volume fraction will barely exceed $20 \%$ in conventional pressure manufacturing processes. 
Table I. Fitted compaction properties for the reclaimed carbon fibre mat. These properties are to be used with equation (I).

\begin{tabular}{llllll}
\hline & & Reclaimed material & Random & Random planar & UD \\
\hline Orientation function & $f$ & 0.192 & & 0.64 & 0 \\
Fibre Young Modulus & $E$ & $240 \mathrm{GPa}$ & & & \\
Power constant & $\mathrm{A}$ & 4.45 & 3 & 5 & \\
\hline
\end{tabular}

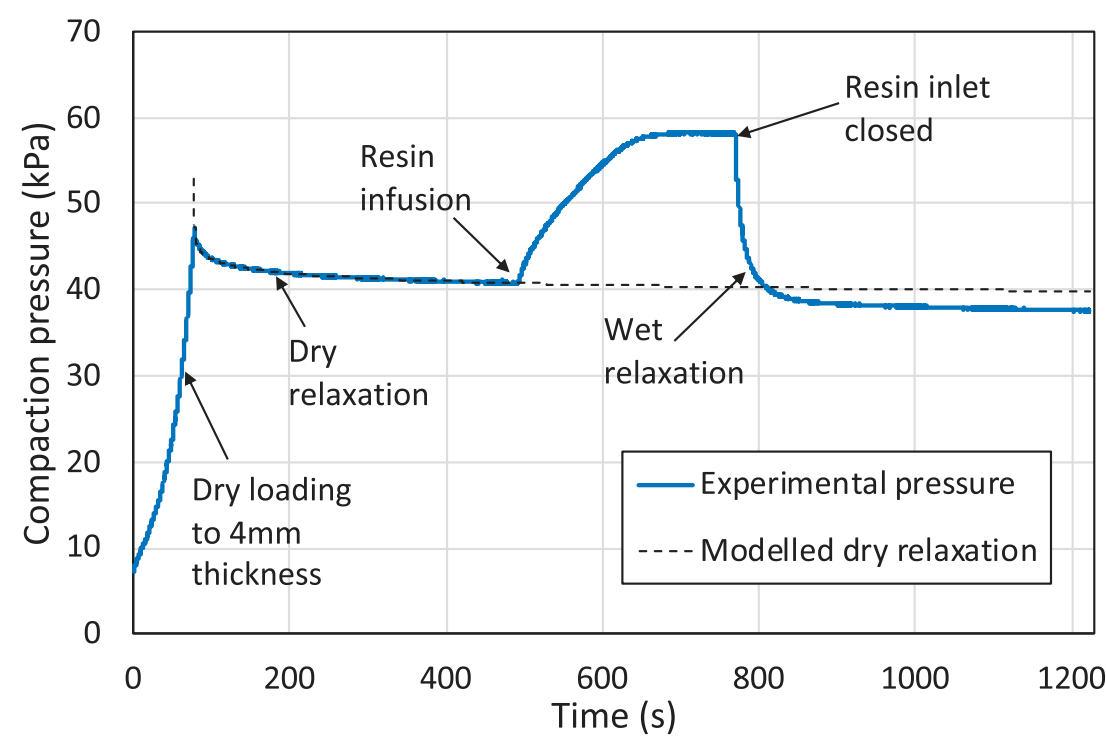

Figure 3. Wet relaxation of a 4-mm thick reclaimed carbon fibre preform. The dry preform is first compressed in a testing machine to the target thickness. A dry relaxation occurs and follows a power law decay. The resin is then injected and the wet relaxation is measured. After $20 \mathrm{~min}$, the difference between the wet and the expected dry response is less than $6 \%$.

resin will act as a lubricant and modify the compaction response. ${ }^{27}$ This modification is very pronounced with unidirectional materials, where the fibre packing and the number of contacts is high. In order to investigate this effect for the reclaimed material used in this study, resin was injected at $100 \mathrm{kPa}$ into a fixed cavity while continuously measuring the compaction force using the methodology described by Kelly et al. $^{27}$ The resulting compaction curve is plotted in Figure 3.

Figure 3 shows that the initial dry relaxation curve follows a power law decay as modelled in literature. ${ }^{27}$ Moreover, after the injection and permeation of the resin in the preform, the wet compaction curve appears slightly shifted downward, which confirms a lubrication effect exists. The final wet compaction force is $37.5 \mathrm{kPa}$ when the dry compaction force would have been $39.8 \mathrm{kPa}$. As a result, for this randomly oriented reclaimed material, with a low fibre volume content, the error is below $6 \%$, and was neglected in the following sections. The fibrebed pressure $P_{f b}$ is assumed to always equate the dry fibrebed pressure.
Infusion fluid. The infusion fluid used in this study was Lyle's brand golden syrup, diluted with $15 \%$ water by weight. The mixture viscosity at room temperature was taken as

$$
\mu=0.1 \mathrm{~Pa} \cdot \mathrm{s}
$$

and was assumed to be constant given the Newtonian behaviour of corn syrup and that all the experiments were carried out at room temperature. Note that in the following, the value of $\mu$ is of little importance as all the magnitudes identified are $\boldsymbol{K} / \mu$. For the sake of clarity and in order to deal with permeabilities, a constant $\mu$ value was used throughout.

\section{RTM setup}

A series of infusion experiments were performed in between rigid flat moulds representative of an RTM process to identify whether the flow front was detected by a change in pressure when a fluid saturates an initially dry fibrebed. The experimental set-up is 
shown in Figure 4. A central injection through a $10-\mathrm{mm}$ inlet was used to introduce the test fluid to the preform. The mould was made of a $20-\mathrm{mm}$ thick aluminium lower platen and an 80-mm thick poly methyl methacrylate (PMMA, also known as acrylic or plexiglass) upper platen. A 4-mm thick 250-mm wide square aluminium spacer was used to maintain a constant laminate thickness during the infusion process, and c-clamps to keep the mould closed.

The infusion trials were performed using a surface mapping sensor and a camera to record the flow front through the transparent upper mould surface. A Tekscan 5101 pressure mapping sensor was placed between the bottom preform ply and the metallic mould surface as shown in Figure 4.

Tekscan sensors are part of a class of tactile pressure sensors that use a piezoresistive material sandwiched between printed conductive circuits where the resistance changes when pressure is applied. ${ }^{28}$ The sensor used in this study was $0.958 \pm 0.008 \mathrm{~mm}$ thick and has a $111.8 \mathrm{~mm}$ square sensing area with measurement points every $2.5 \mathrm{~mm}$. The sensor was equilibrated and calibrated according to the manufacturer's recommendations. The sensor measured pressure to within $6 \%$ of a universal testing machine in the pressure range of this study.

\section{Procedure}

The preform was made by stacking 5 layers of reclaimed material or 10 layers of unidirectional materials that were $180-\mathrm{mm}$ in diameter. A $12-\mathrm{mm}$ diameter hole was punched in the centre of the preform to ensure a fullydeveloped 2D flow occurred in-plane. The preform was intentionally cut smaller than the spacer to ensure a uniform vented pressure was present at the perimeter.

For each test, a positive relative pressure of $160 \mathrm{kPa}$ was applied to the fluid using a pressure pot connected to a central air supply having a maximum pressure of $700 \mathrm{kPa}$. The camera and pressure sensor acquisition was started at time $t=0$ when the fluid reached the preform. A synchronised acquisition was performed on both the camera and the pressure sensor every $5 \mathrm{~s}$ throughout the infusion.

\section{Theoretical and numerical methods}

\section{Flow modelling}

This section models the flow front evolution and resin pressure field in the case of the central infusion performed in the experiment.

Geometry. The 2D geometry is represented in Figure 5, with the unknown parameters being the flow front position and the resin pressure field $P(x, y)$. Atmospheric pressure was applied at the preform perimeter.

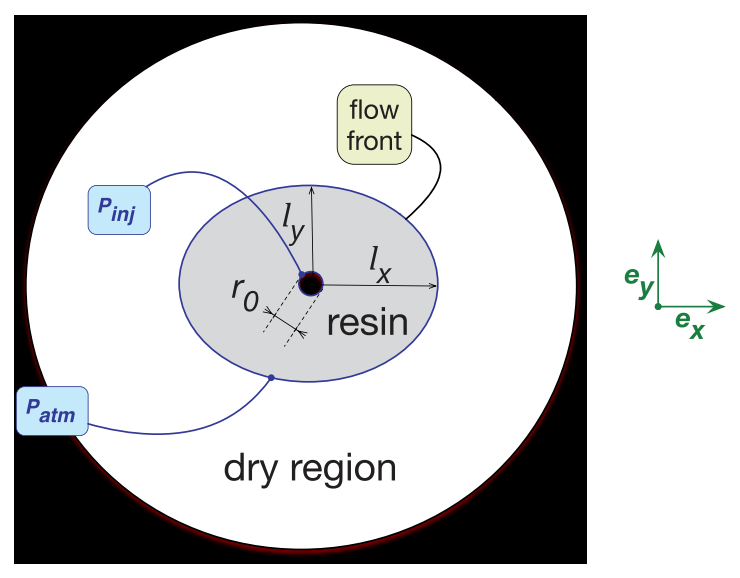

Figure 5. 2-Dimensional geometry considered in the infusion model. A central hole was punched through the preform. The injection pressure is thus imposed on this hole perimeter. The outside perimeter of the preform is vented to atmospheric pressure.
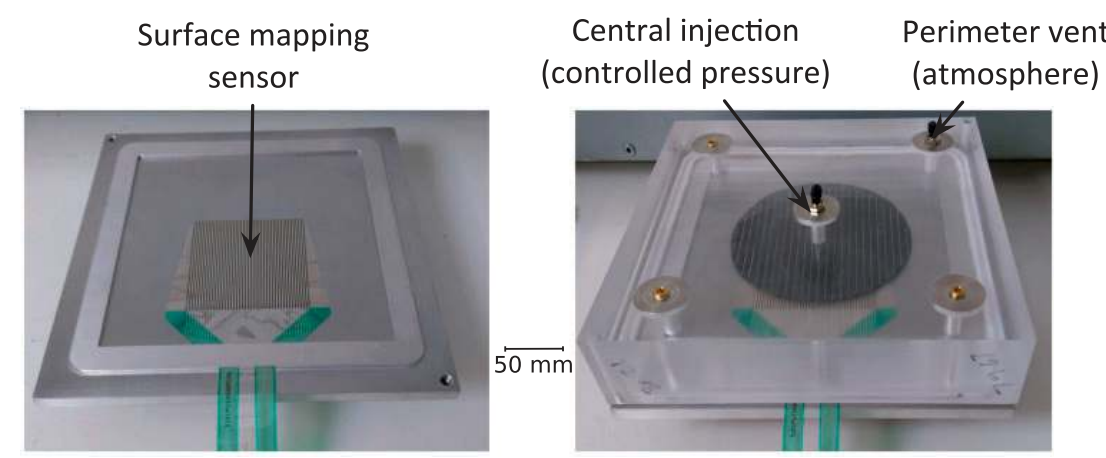

Figure 4. Experimental infusion setup. During an experiment, the transparent lid allows for camera recording through the upper platen, while simultaneously on the lower platen, the pressure mapping sensor acquires the preform (black circle) pressure field. 
Behaviour. Following classical modelling of infusion processes, a Darcy's behaviour is assumed in the preform. ${ }^{13,14}$ The apparent velocity $\boldsymbol{v}$ is

$$
\boldsymbol{v}=-\frac{\boldsymbol{K}}{\mu} \nabla P
$$

where $\nabla$ is the spatial derivative operator and $\boldsymbol{K}$ is the in-plane permeability tensor of the preform. Because the orientation of both the UD material and the reclaimed material are known and aligned with the $\boldsymbol{e}_{x}$ direction, the permeability principal directions are $\boldsymbol{e}_{x}$ and $\boldsymbol{e}_{y}$. Thus, the permeability tensor is diagonal and can be written as

$$
\boldsymbol{K}=\left[\begin{array}{cc}
k_{x} & 0 \\
0 & k_{y}
\end{array}\right]_{\left(e_{x}, e_{y}\right)}
$$

where $k_{x}$ is the longitudinal and $k_{y}$ the transverse inplane permeabilities.

Assuming incompressible resin, the continuity equation simplifies to

$$
\nabla \cdot \boldsymbol{v}=0
$$

which gives, with equation (5) the classical constitutive equation for the pressure field ${ }^{13,14}$

$$
\nabla \cdot\left(\frac{\boldsymbol{K}}{\mu} \nabla P\right)=0
$$

Boundary conditions. During infusion, the pressure was kept constant in the resin pot. Neglecting pressures loss in the inlet lines, the relative injection pressure $P_{i n j}$ was applied to the hole circumference.

On the outer boundary, two equivalent approaches can be assumed:

1. The viscosity of the air being very low compared to that of the resin (about three orders of magnitudes lower), and the pressure can be considered homogeneous in the dry region. Therefore, atmospheric pressure was assumed at the flow front position. The problem is solved in the saturated region only, which grows with the flow front motion.

2. A Darcy flow was assumed for the air in the dry region, resulting in a two-phase flow in a fixed geometry (the whole preform) with a moving interface. This is the assumption made in the numerical simulation described in section Numerical implementation.

Analytical solution. Under the assumption of a uniform permeability tensor in the preform, the Darcy flow problem can be solved analytically. This analytical model will be useful for characterising effective homogeneous permeabilities of the preform.

To account for the permeability anisotropy, a reference frame was used. ${ }^{14}$ Details are given in Appendix 1 Radial Darcy flow. The pressure is described as

$$
P=P_{i n j} \times \frac{\ln (r)-\ln (l)}{\ln \left(\sqrt{\beta} r_{0}\right)-\ln (l)}
$$

where $r=\sqrt{x^{2}+\beta^{2} y^{2}}$ is the radial position in the reference frame and $\beta=\sqrt{k_{x} / k_{y}}$ is the permeability anisotropy ratio. $\beta$ is the ratio of the major and minor axis of the ellipse flow front. The flow front position $l$ along the major principal $x$ direction evolves as

$$
l(t)=r_{0} \sqrt{\frac{\beta t / \gamma}{W\left(\frac{t}{\gamma e}\right)}}
$$

where $W$ is the Lambert $\mathrm{W}$ function, $e$ the Euler number and

$$
\gamma=\frac{\mu \beta r_{0}^{2}}{4 k_{x} P_{i n j}}
$$

is the characteristic infusion time.

\section{Direct numerical simulation}

Analytical models assuming uniform permeabilities are not meant to capture defects induced by local material variability. Variability observed in fibre volume fractions has been shown to influence the flow front. ${ }^{8}$ In light of the likelihood of variability-induced defects in the reclaimed fibre mat flow front, a direct numerical simulation framework is proposed in this section where the pressure mapping sensor data are used as an input to account for local material variability.

Permeability mapping. The radial infusion model presented in section Flow modelling is used again here; however, permeability of the preform $\boldsymbol{K}$ is no longer uniform but depends on the position $(x, y)$. The permeability at each point was assessed from the fibrebed pressure map $P_{f b}$ which can directly be obtained from the pressure mapping sensor prior to infusion. Using the compaction behaviour characterised in section Fibrebed compaction curve the fibre volume fraction map was obtained as follows

$$
V_{f}(x, y)=\frac{P_{f b}^{\frac{1}{4}}(x, y)}{\left(E f^{4}\right)^{\frac{1}{4}}}
$$

The permeability field in $x$ and $y$ was obtained using the classical Kozeny-Carman assumption given by 
equation (2). The constant tensor $\boldsymbol{C}$ in equation (2) was identified using the effective permeabilities characterised off-line in section Material characterisation. The two principal effective permeabilities $k_{x}^{0}$ and $k_{y}^{0}$ obtained in that section corresponds to an average compaction state inferred from the mould gap thickness as an average pressure $\left\langle P_{f b}\right\rangle=17.1 \mathrm{kPa}$ and a corresponding average volume fraction $V_{f}^{0}=0.109$.

Numerical implementation. The Darcy flow problem in equation (8) is a Laplace equation. It was solved numerically using a finite element method and an open-source partial differential equation solver: FreeFEM $++{ }^{29}$ The FreeFEM ++ script is available under open-source license, available to download from the link provided after the conclusion section. A P1 interpolation was used for the pressure field. As stated in the previous section, the problem was solved over the whole preform. The flow front was tracked with a levelset method. ${ }^{30}$ The resin volume fraction $\varphi$, which is also described with a P1 interpolation, was obtained from the levelset field $\psi$ using a classical smoothed step function

$$
\phi=\frac{1}{2} \frac{\psi}{\sqrt{\psi^{2}+h^{2} \times|\nabla \psi|^{2}}}+\frac{1}{2}
$$

where $h$ is the interface thickness, ${ }^{31}$ set to $0.1 \mathrm{~mm}$. The viscosity was set according to the resin volume fraction either to that of the resin $\mu=0.1 \mathrm{~Pa} \cdot \mathrm{s}$ in the wet region or to that of the air $\mu=0.001 \mathrm{~Pa} \cdot \mathrm{s}$ in the dry region. The permeability tensor at the pressure sensor cell locations was obtained from the fiber bed pressure measurements $P_{f b}$ using equations (1) and (2) before linear interpolation and projection onto the finite element mesh to obtain the permeability tensor field. The pressure mapping sensor does not cover the entire preform, therefore the permeability for the uncovered area was set to the effective permeability identified in section Material characterisation. The results presented hereunder were cropped to the pressure sensor area.

The time integration followed a standard iterative procedure with constant time steps of $1 \mathrm{~s}$. At each time iteration:

1. The Darcy flow constitutive equation (8) was solved and gave the pressure and apparent velocity fields.

2. The levelset was convected using a CharacteristicsGalerkin method. ${ }^{29}$ A levelset inward flux was also imposed on the inlet hole to prevent artefacts, like levelset sign change, at the vicinity of this inlet boundary.

3. The mesh was refined at the vicinity of the interface to keep a fine description of the flow front morphology, using the FreeFem ++ built-in adaptive remeshing. ${ }^{29}$

4. The fields were projected onto the new mesh before updating the phase field and viscosities.

5. The levelset was reinitialised as detailed by El-haddad et al. ${ }^{31}$ to ensure that the gradient norm remained at unity.

In order to prevent numerical singularities, the initial flow front position was set at a distance of $l_{0}=1.3 * r_{0}=7.8 \mathrm{~mm}$ instead of $r_{0}=6 \mathrm{~mm}$. This initial shift is still small compared to the preform dimension of $180 \mathrm{~mm}$. Accordingly, the simulations started at a positive physical time.

\section{Data processing}

The procedure to analyse both the image and pressure sensor experimental data is described in this section.

Camera images. Each grey scale image frame (one taken every $5 \mathrm{~s}$ ) was processed independently. The processing was performed in MATLAB using the built-in image processing toolbox. The first frame, which consisted of only the dry preform, was used as the background and subtracted from every subsequent frame. A Gaussian filter with a standard deviation of 5 pixels was applied before the image was thresholded to obtain a mask representing the saturated region. A successive dilatation and erosion of 20 pixels was applied to the image to eliminate artefacts, such as the inlet tubing that was visible in the image. An ellipse was then fit to the obtained image mask, using the standard second moments technique built in MATLAB, to determine the major and minor axis of the flow front.

Experimental pressure field. The pressure map obtained with the pressure mapping sensor was analysed at each acquisition time step independently. The initial frame, before resin infusion, represented the fibrebed compaction pressure after mould closing and is called the fiber bed pressure map, $P_{f b}$. It is represented in Figure 6 . The total measured pressure $P_{t o t}$ during the infusion phase is the sum of this fibrebed pressure and the resin pressure $P .^{13}$ The fibrebed pressure was considered constant throughout the process and thus any lubrication effects ${ }^{25,32}$ were neglected. The fibrebed pressure $P_{f b}$ can thus be directly subtracted to the total pressure $P_{\text {tot }}$ to give the resin pressure map at each time

$$
P=P_{t o t}-P_{f b}
$$

A pragmatic approach to obtain the flow front position is to threshold the resin pressure field $P$ directly. 


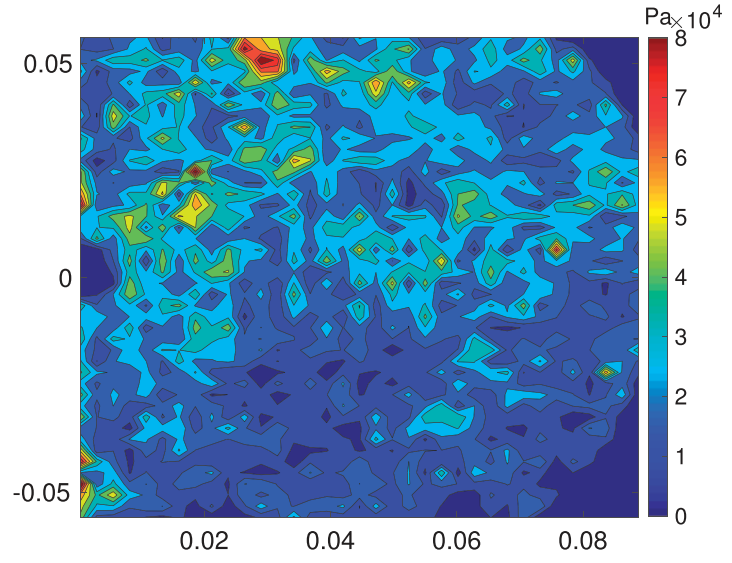

Figure 6. Fiber bed compaction pressure map $P_{f b}$ measured by the pressure sensor prior to infusion for the reclaimed fibre material. The pressure distribution is non-uniform.

Radial streamline analysis. A more theoretical approach to determining the flow front position was found using the streamline method described by Di Fratta et al. ${ }^{20}$ In the case of a central injection of a circular preform, the streamlines follow a straight path from the central injection point to the preform edge. Thus, the procedure is called radial streamline approach. A slight error may arise in the case of non-uniform permeability resulting from non-straight streamlines. In this radial streamline approach, along each of these streamlines, if the material was to be uniform, the pressure field should follow equation (9). Thus, the pressure versus the logarithm of the distance from the central injection point fits a straight line. This straight line crosses the pressure $P=0$ at the flow front position.

The radial streamline approach takes advantage of the quantitative pressure measurements obtained from the pressure mapping sensor instead of binary pressure thresholding at the vicinity of the flow front. Therefore, it determines the flow front measurement more accurately than the thresholding method which has a spatial accuracy equal to the pressure cell spacing.

Permeability characterisation. The flow front detected with the camera was used to characterise the permeabilities $k_{x}$ and $k_{y}$. The major and minor semi-axis of the ellipse versus time were fitted to the analytical model given in equation (10).

The experimental pressure field versus time, obtained with the pressure mapping sensor $P_{\text {exp }}$, was also used to characterise the permeability of the preform. Assuming a uniform effective anisotropic homogeneous permeability, given by equation (6), the pressure field $P_{\bmod }$ can be modelled analytically as developed in section Flow modelling. Using a classical inverse method, the longitudinal and transverse in- plane permeabilities $k_{x}$ and $k_{y}$ can be inferred by minimising the difference between $P_{\text {mod }}$ and $P_{\text {exp }}$

$$
\min _{\left(k_{x}, k_{y}\right)} \sum_{t, x, y}\left[P_{\bmod }(x, y, t)-P_{\text {exp }}(x, y, t)\right]^{2}
$$

The minimisation was performed using the built-in simplex method in MATLAB.

\section{Results and discussion}

\section{Flow front detection}

The resin pressure map obtained from the experiments was used to detect the flow front position. A first pragmatic method consisted of thresholding the pressure field to $7 \%$ of the injection pressure to detect the flow front. Additionally, the radial streamline approach, based on the method by Di Fratta et al. ${ }^{20}$ described in section Radial streamline analysis was also applied to the resin pressure map. The pressure field was analysed along streamlines emanating from the central injection point in the radial streamline analysis. An example of a pressure along a streamline to the central injection point is shown in Figure 7. Plotting the pressure as a function of the logarithm of the distance gives a linear line. Extrapolating this fit line to intersect $P=0$ gives the flow front distance.

A comparison between the pressure map thresholding and radial streamline pressure approaches to identifying the flow front position are shown in Figure 8. The flow front obtained with the camera is also included. A video containing the full sequence of time frames is provided as supplementary material with this article.

The flow fronts obtained with both pressure mapping methods agrees with the flow front found by the camera images for the reclaimed mat material, as shown in Figure 8. The radial streamline approach appears more robust to identifying the full flow front, whereas the pressure thresholding method introduces some abnormalities due to noise and artefacts at low pressures. The thresholding methods only analyses the vicinity of $P=0$ whereas the radial streamline approach takes advantage of the full pressure field. A particular strength of the radial streamline pressure approach was found later in the injection, when the wet area had increased and accordingly the pressure gradient had decreased, resulting in imprecise flow fronts by the pressure thresholding method. Even for longer times (at $t=595 \mathrm{~s}$ ), the error between the flow front detected by the camera and the pressure sensor using the radial streamline approach is less than $5 \%$.

In the case of the unidirectional virgin material (left plot in Figure 8), the flow front position found by the 


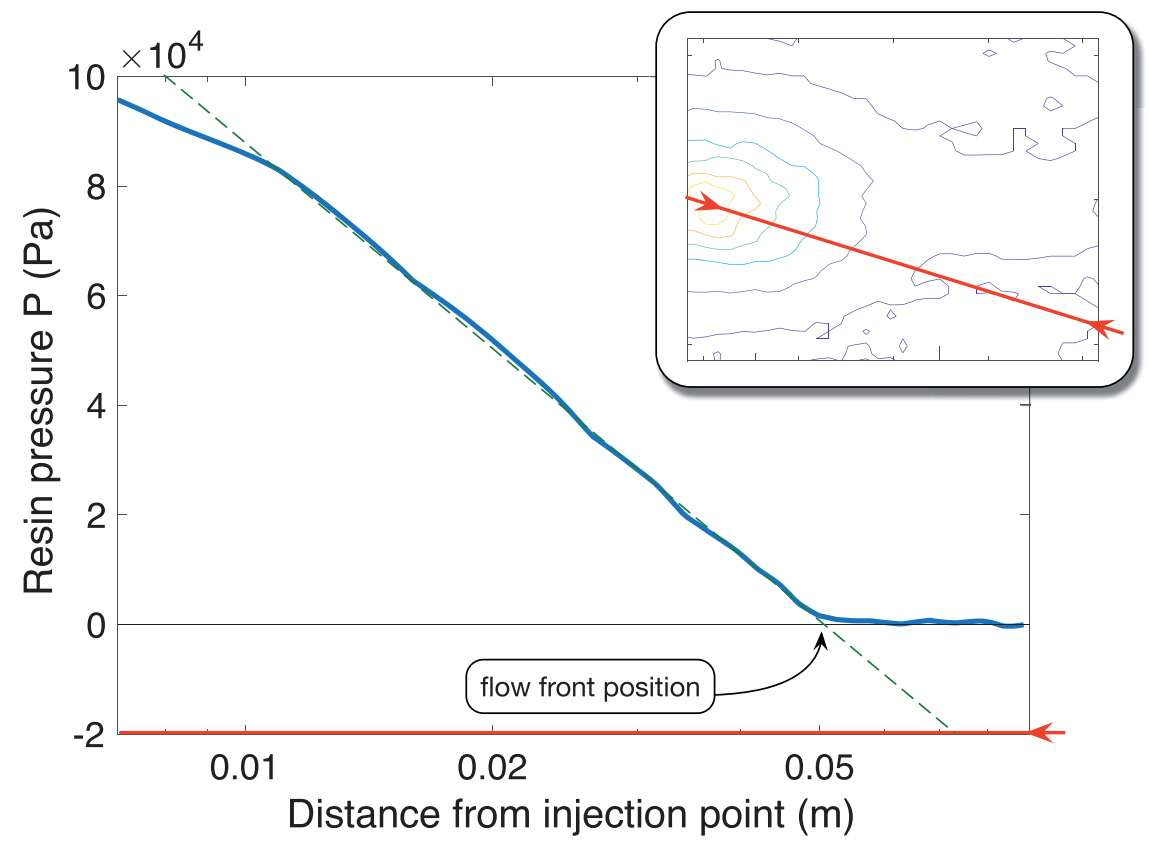

Figure 7. Resin pressure versus distance from the central injection point along one particular streamline. In the radial streamline analysis, the flow front position is determined by fitting a line through the semi-log plot and extrapolating it to $P=0$.

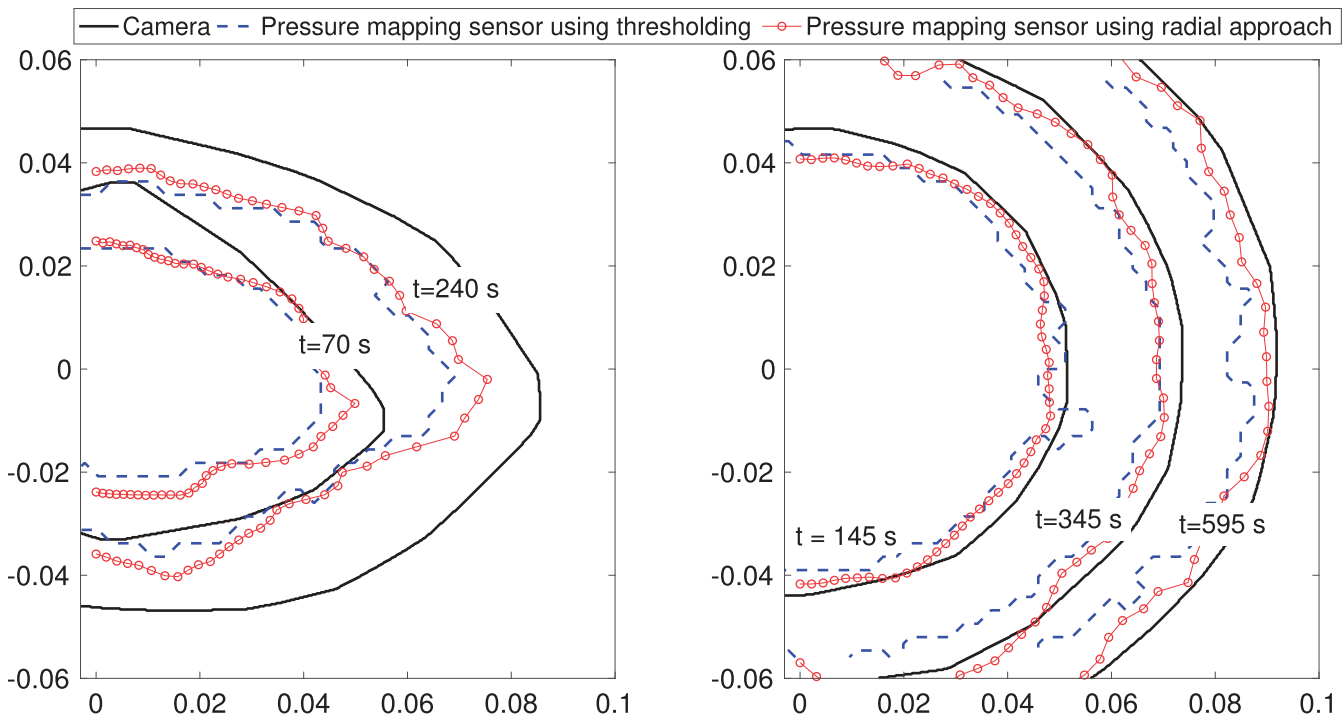

Figure 8. Flow front detection for the unidirectional virgin material (left) and reclaimed mat material (right) using pressure mapping data and camera images.

pressure mapping approaches does not agree with the camera. The flow front by image analysis is always leading the flow front obtained with the pressure sensor (using both thresholding or radial streamline approach). Two explanations are suggested:

1. The capillary effects are neglected in the analysis. In the case of tows with high fibre volume content, the capillary effect cannot be neglected in the vicinity of the resin front. ${ }^{33}$ The partially saturated zone, which is invisible to the pressure sensor, is more pronounced in the unidirectional materials and less pronounced in the reclaimed fibre mat.

2. The reclaimed fibre mat compaction pressure was assumed constant throughout the injection. In particular, lubrication effects were neglected. In the case of unidirectional materials, with a higher number of parallel fibre contacts, the lubrication effect may 
result in a wet compaction pressure smaller than the assumed fibrebed compaction pressure $P_{f b}$ in equation (14).

\section{Material characterisation}

The pressure mapping sensor data were used to characterise the preform permeability as described in section Permeability characterisation. This was performed successively with a virgin unidirectional preform and a reclaimed material preform. The fitted pressure field at time $t=145 \mathrm{~s}$ is shown in Figure 9 along with the experimental measured pressure field for representative tests of each material.

The direct analytical model predicts smooth elliptical isobars whereas the experimental flow front measured by the sensor is more jagged. The obtained longitudinal and transverse permeabilities are reported in Table 2. Within the same experiment, the ellipse minor and major axis versus time were obtained from the image processing allowed for a camera-based characterisation. An inverse method was used to fit the analytical flow front evolution given by equation (10) to these experimental axes. The fitting is shown in Figure 10. The obtained permeabilities are given in Table 2.

For the reclaimed material, the permeabilities obtained by both methods are consistent within $8 \%$. The random fibre orientation in the reclaimed material is reflected by permeability ratio $\beta$ that is close to 1 . The highly aligned virgin unidirectional material, made out of oriented tows, is reflected by a large permeability anisotropy ratio $\sqrt{k_{x} / k_{y}}$. The flow front lag observed in Figure 8 resulted in a discrepancy of as much as $15 \%$ in the permeabilities identified using the camera and pressure mapping sensor methods (Table 2). Overall, the permeabilities identified with the camera are higher than those obtained with the pressure sensor. This was attributed to the partially saturated zone that is more predominant in the case of the unidirectional material tested.

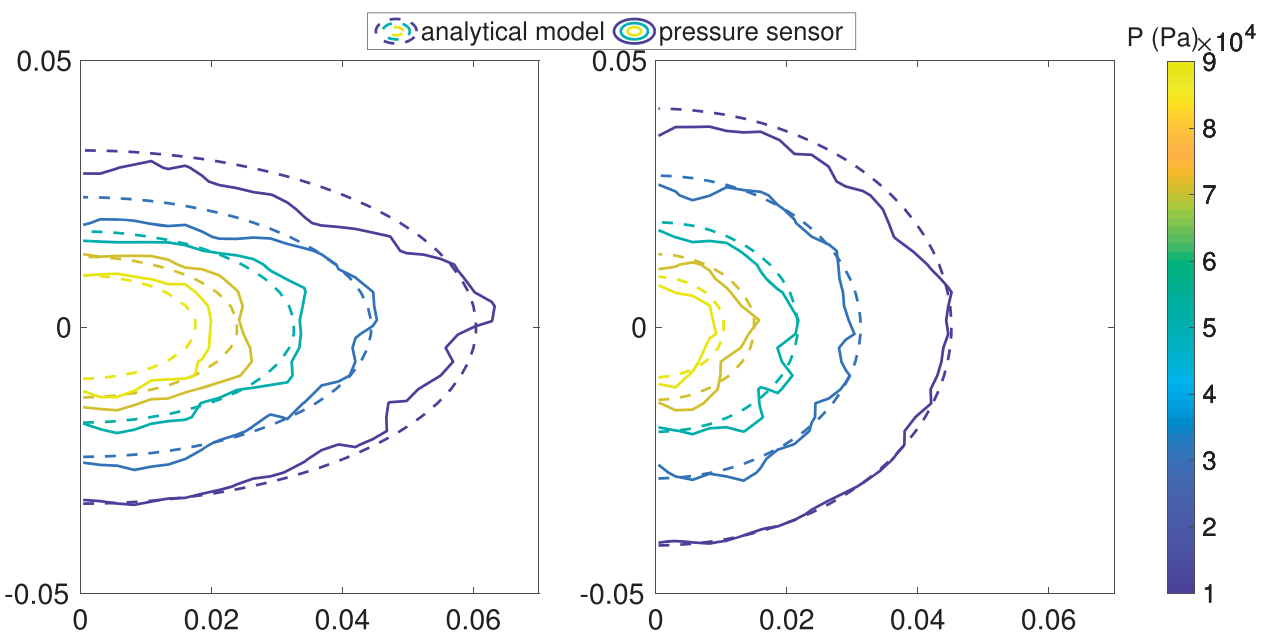

Figure 9. Resin pressure map measured using the pressure sensor and predicted using the analytical model at time step $t=145 \mathrm{~s}$ for the unidirectional virgin material (left) and reclaimed material (right). The longitudinal and transverse permeabilities (respectively $k_{x}$ and $k_{y}$ ) were obtained by fitting these two fields over the entire duration of the injection.

Table 2. Permeability characterisation using the pressure map and the camera ellipse fit for the reclaimed and UD material. The two methods are consistent. The unidirectional virgin material has a much better fibre alignment resulting in a greater permeability anisotropy.

\begin{tabular}{|c|c|c|c|c|}
\hline & \multicolumn{2}{|l|}{ UD } & \multicolumn{2}{|l|}{ Reclaimed } \\
\hline & Pressure & Camera & Pressure & Camera \\
\hline$k_{x}\left[m^{2}\right]$ & $13.8 \times 10^{-12}$ & $14.1 \times 10^{-12}$ & $11.2 \times 10^{-12}$ & $12.2 \times 10^{-12}$ \\
\hline$k_{y}\left[\mathrm{~m}^{2}\right]$ & $3.83 \times 10^{-12}$ & $4.47 \times 10^{-12}$ & $9.29 \times 10^{-12}$ & $9.17 \times 10^{-12}$ \\
\hline$\beta$ & 1.90 & 1.77 & I.II & 1.15 \\
\hline
\end{tabular}




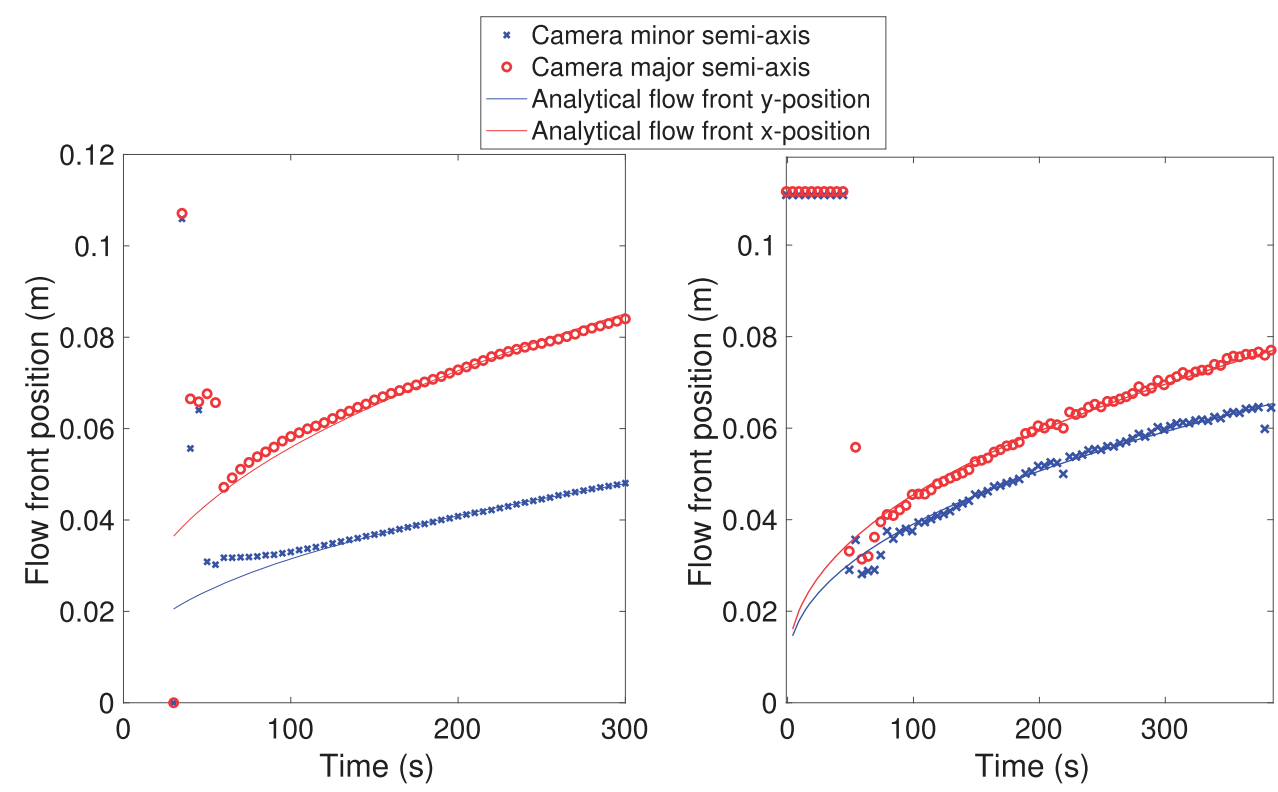

Figure 10. Evolution of minor and major semi-axis of the elliptic resin front versus time for the unidirectional virgin material (left) and the reclaimed material (right). Comparison between the camera measurement and the analytical model. There is an artefact in the first experimental data points due to the presence of the resin inlet, covering the first few millimetres of the flow front.
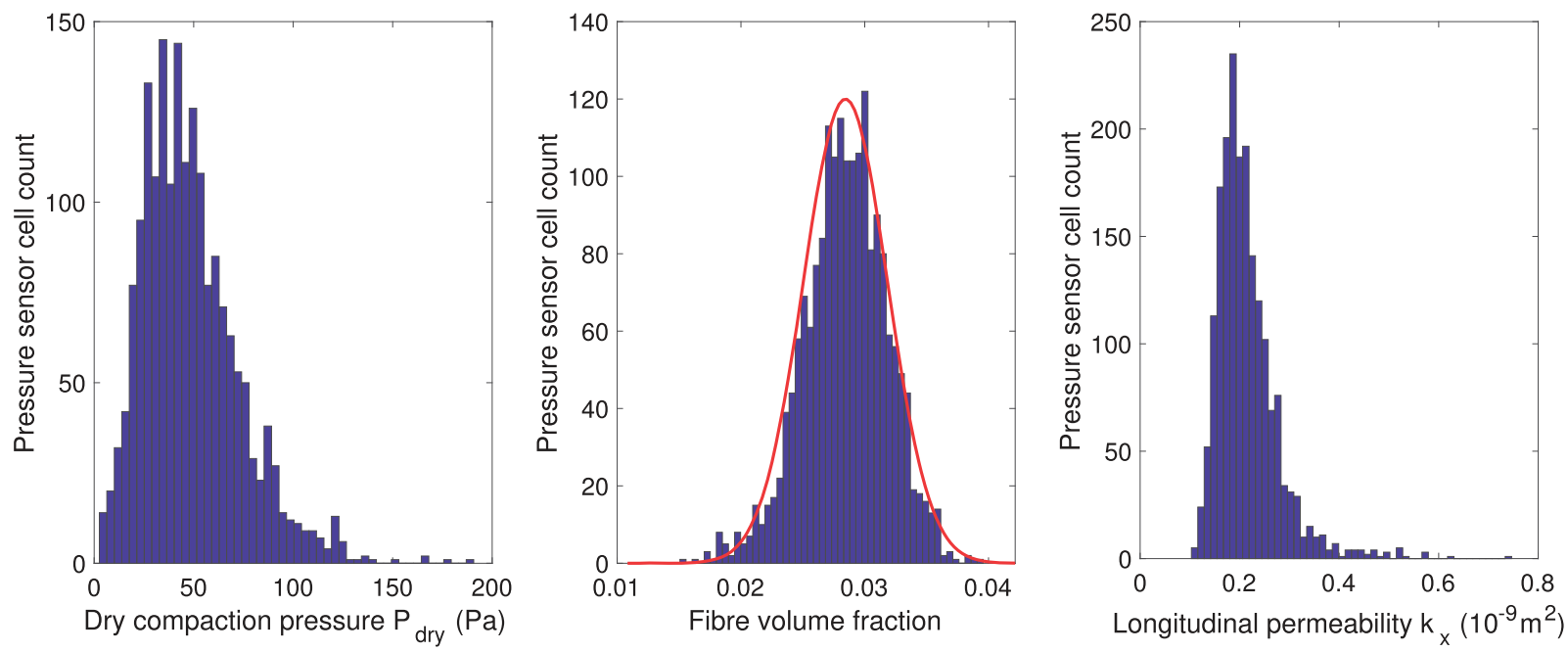

Figure II. The permeability used in the direct numerical simulation is obtained from the initial dry fibrebed pressure map using the compaction curve and Kozeny-Carman assumption. The distribution of fibrebed compaction pressure (left), fibre volume fraction (middle), and longitudinal permeability (right) is presented here in the form of histograms. Only the fibre volume fraction variability follows a normal distribution.

The permeability values obtained from the camera images are based on the flow front position observed through the upper mould surface. On the other hand, the permeability data obtained from the pressure sensor are using the whole pressure field of the preform during infusion. This class of pressure sensing technology shows potential for scale-up to industrial applications where transparent moulds or inserts might be impractical, due to process temperature limitations or leakfree tooling requirements.

\section{Direct numerical simulation}

This section presents the results of the direct numerical simulation of the infusion problem. As detailed in the method Direct numerical simulation, the simulation is based on the input provided by the pressure sensor prior to infusion (Figure 6).

A variability analysis was performed over the pressure sensor and is presented as histograms in Figure 11 for one single reclaimed preform. The dry fibrebed 
compaction pressure is converted to fibre volume fraction using the compaction law in equation (1). Even though the compaction pressure variability is not normally distributed, because the compaction is nonlinear, the volume fraction variability appears to be normally distributed, as shown on the middle histogram in Figure 11. This might be expected given the reclaiming process used to produce the random fibre mat. The fibre volume fraction map was then converted to a permeability map using the Kozeny-Carman law in equation (2). The non-linear Kozeny-Carman equation predicts a non-normal permeability distribution in the preform. The permeability variability within the reclaimed fibre preform is high, with a coefficient of variation of $45 \%$. The direct numerical simulation approach takes this variability into account in the simulation.

The experimental flow front obtained with the pressure sensor (using the radial streamline approach) are compared with flow fronts computed by the direct numerical simulation of the reclaimed material preform, in Figure 12. The material variability results in a flow front position which deviates from the elliptical flow front predicted by a homogeneous permeability. The direct numerical simulation takes into account this initial preform variability and is able to track the experimental flow front more accurately. In this particular case, the non-symmetry observed in Figure 6 exhibits a higher fibre-bed compaction pressure in the upper half, and is mapped to a lower permeability in this half of the preform. Accordingly, in Figure 12, the direct numerical simulation of the flow front is asymmetric, which cannot be captured by the analytical homogeneous prediction.

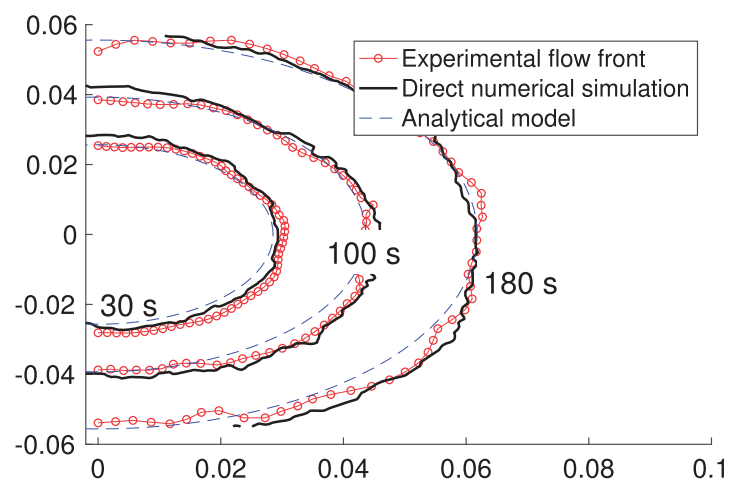

Figure 12. Comparison of the flow fronts for reclaimed material obtained experimentally with the pressure sensor, numerically using the direct numerical simulation and analytically considering a uniform permeability. The material variability is reflected in variability in the flow front positions, which is well predicted with the direct numerical simulation.

\section{Conclusion}

A pressure mapping sensor was used to both initialise an infusion simulation and subsequently track the flow front in an RTM process consisting of preforms made using reclaimed fibre mats and virgin unidirectional materials. The pressure sensor provided a large amount of data as it contained almost 2000 measurement points in an $11 \mathrm{~cm} \times 11 \mathrm{~cm}$ area. The full field pressure data were used in three ways.

First, the pressure data were used to track the resin flow front during infusion and compared with synchronised camera observations through a transparent mould. Good agreement was observed for the reclaimed material. However, the flow-front detected for the unidirectional material was consistently lagging the camera position, as capillary and lubrication effects were more pronounced. The camera detects the front of the unsaturated zone whereas the pressure sensor detects the front of the saturated zone.

Second, the full experimental pressure field was used with an inverse method to characterise the in-plane permeability tensor. The longitudinal and transverse reclaimed material permeabilities were within $10 \%$ of the values characterised by the camera. The flow-front disparity in the unidirectional material was carried forward to the permeability tensor.

Finally, the initial pressure map measured after mould closure was used to predict volume fraction and permeability maps using the compaction behaviour and Kozeny-Carman equation. The permeability was used as an input in direct numerical simulation of a subsequent infusion step. The simulation matched the experimental flow-front which differs from the predicted flow front using a homogeneous permeability assumption. This direct numerical simulation accounts for any local material variability which is inherent to composite materials and was more pronounced in the reclaimed random fibres mats used in this study.

Overall, the methodology presented here demonstrates how in-process measurement prior to injection can be used as an input for accurate simulation of the flow front. In an industrial framework, such an approach could be integrated in a closed loop control of the RTM process. Variability-induced defects could be mitigated by adjusting process parameters, such as injection gates or vents pressure, to maximise filling success for every preform. Additional considerations around sensor integration into production tooling will be required before full scale-up to industrial applications is realised.

\section{Acknowledgements}

The authors acknowledge Miss Lina Zschenderlein for her help with the experimental campaign. The authors thank Dr Jonathan Belnoue for kindly reviewing the manuscript. 


\section{Declaration of Conflicting Interests}

The author(s) declared no potential conflicts of interest with respect to the research, authorship, and/or publication of this article.

\section{Funding}

The author(s) disclosed receipt of the following financial support for the research, authorship, and/or publication of this article: The authors acknowledge financial support from the Institute for Advanced Studies at the University of Bristol for a 3-month Benjamin Meaker Visiting Fellowship for Dr Arthur Levy. The reclaimed fibre material was kindly donated by the SGL group.

\section{ORCID iD}

Arthur Levy (D) https://orcid.org/0000-0001-9221-6850

\section{Supplemental material}

Supporting data are available from the University of Bristol research data repository at: https://data.bris.ac.uk/data/dataset/3d44jy10nv8h52cbtld95kjfev

\section{References}

1. Kratz J, Low, Yi S and Fox B. Resource-friendly carbon fiber composites: combining production waste with virgin feedstock. Adv Manufact Polym Compos Sci 2017; 3: 121-129.

2. Advani SG and Hsiao K. Manufacturing techniques for polymer matrix composites (PMCs). Cambridge, UK: Woodhead Publishing, series in edition, 2012.

3. Potter K. Resin transfer moulding. Dordrecht, The Netherlands: Springer Science \& Business Media, 1997.

4. Hammami A, Gauvin R and Trochu F. Modeling the edge effect in liquid composites molding. Compos $A$ Appl Sci Manufact 1998; 29: 603-609.

5. Bickerton S and Advani SG. Characterization and modeling of race-tracking in liquid composite molding processes. Compos Sci Technol 1999; 59: 2215-2229.

6. Siddig NA, Binetruy C, Syerko E, et al. A new methodology for race-tracking detection and criticality in resin transfer molding process using pressure sensors. $J$ Compos Mater 2018; 52(29): 4087-4103. DOI: 10.1177/0021998318774829.

7. Caglar B, Salvatori D, Sozer EM, et al. In-plane permeability distribution mapping of isotropic mats using flow front detection. Compos A 2018; 113: 275-286.

8. Endruweit A, Harper LT, Turner TA, et al. Composites: Part A random discontinuous carbon fibre preforms: permeability modelling and resin injection simulation. Compos A Appl Sci Manufact 2008; 39: 1660-1669.

9. Walbran WA, Bickerton S and Kelly PA. Measurements of normal stress distributions experienced by rigid liquid composite moulding tools. Compos A Appl Sci Manufact 2009; 40: 1119-1133.

10. Endruweit A, Harper LT, Turner TA, et al. Random discontinuous carbon fiber preforms: experimental permeability characterization and local modeling. Polym Compos 2010; 31: 569.
11. Toll S and Manson J-AE. Elastic compression of a fiber network. J Appl Mech 1995; 62: 223-226.

12. Gutowski TG, Morigaki T and Cai Z. The consolidation of laminate composites Iof. J Compos Mater 1987; 21: $172-188$.

13. Gutowski TG. Advanced composites manufacturing. New York, Chichester, Weinheim, Singapore, Toronto: John Wiley \& Sons, 1997.

14. Advani SG and Sozer EM. Process modeling in composites manufacturing. Boca Raton, FL: CRC Press, 2011.

15. Gutowski TG, Cai Z, Bauer S, et al. Consolidation experiments for laminate composites. J Compos Mater 1987; 21: 650-669.

16. Gebart BR. Permeability of unidirectional reinforcements for RTM. J Compos Mater 1992; 26: 1100-1133.

17. Vernet N, Ruiz E, Advani S, et al. Experimental determination of the permeability of engineering textiles: Benchmark ii. Compos A Appl Sci Manufact 2014; 61: 172-184.

18. May D, Aktas A, Advani SG, et al. In-plane permeability characterization of engineering textiles based on radial flow experiments: a benchmark exercise. Compos $A$ Appl Sci Manufact 2019; 121: 100-114.

19. Konstantopoulos S, Fauster E and Schledjewski R. Monitoring the production of FRP composites: a review of in-line sensing methods. eXPRESS Polym Lett 2014; 8: 823-840.

20. Di Fratta C, Klunker F and Ermanni P. Composites: Part A, A methodology for flow-front estimation in LCM processes based on pressure sensors. Compos $A$ 2013; 47: 1-11.

21. Sozer EM, Bickerton S and Advani SG. On-line strategic control of liquid composite mould filling process. Compos A Appl Sci Manufact 2000; 31: 1383-1394.

22. Nielsen DR and Pitchumani R. Closed-loop flow control in resin transfer molding using real-time numerical process simulations. Compos Sci Technol 2002; 62(2): 283-298.

23. Hsiao K-T and Advani SG. Flow sensing and control strategies to address race-tracking disturbances in resin transfer molding. part i: design and algorithm development. Compos A Appl Sci Manufact 2004; 35: 1149-1159.

24. Liu Q, Parnas RS and Giffard HS. New set-up for inplane permeability measurement. Compos A Appl Sci Manufact 2007; 38: 954-962.

25. Okonkwo K, Simacek P, Advani SG, et al. Characterization of $3 \mathrm{D}$ fiber preform permeability tensor in radial flow using an inverse algorithm based on sensors and simulation. Compos A Appl Sci Manufact 2011; 42: 1283-1292.

26. Walbran WA, Verleye B, Bickerton S, et al. Prediction and experimental verification of normal stress distributions on mould tools during liquid composite moulding. Compos A Appl Sci Manufact 2012; 43: 138-149.

27. Kelly PA, Umer R and Bickerton S. Viscoelastic response of dry and wet fibrous materials during infusion processes. Compos A Appl Sci Manufact 2006; 37: 868-873.

28. Tekscan. The difference between film $\&$ tactile sensors for pressure measurements. Mach Design 2018. 
29. Hecht F. New development in FreeFem++. J Num Math 2012; 20: 251-265.

30. Sethian JA. Level set methods and fast marching methods: evolving interfaces in computational geometry, fluid mechanics, computer vision, and materials science. Cambridge, UK: Cambridge University Press, 1999.

31. El-haddad M, Hecht F and Sayah T. Interface transport scheme of a two-phase flow by the method of characteristics. Int J Num Meth Fluids 2016; 83: 513-543.

32. Robitaille F and Gauvin R. Compaction of textile reinforcements for composites manufacturing I: review of experimental results. Polym Compos 1998; 19: 198-216.

33. Bréard J, Henzel Y, Trochu F, et al. Analysis of dynamic flows through porous media. Part I: Comparison between saturated and unsaturated flows in fibrous reinforcements. Polym Compos 2003; 24: 391-408.

34. Wang JT, $\mathrm{Wu} \mathrm{CH}$ and Lee JL. In-plane permeability measurement and analysis in liquid composite molding. Polym Compos 1994; 15: 278-288.

35. Barry DA, Culligan-Hensley PJ and Barry SJ. Real values of the w-function. ACM Trans Math Software 1995; 21: 161-171.

\section{Appendix I}

\section{Radial Darcy flow}

This appendix describes the analytical resolution of the Darcy flow with a central injection point as modelled in section Flow modelling.

\section{Anisotropy handling}

The anisotropy of the permeability tensor in composite materials (usually $k_{x}>k_{y}$ ) leads to a non-radial, elliptical flow problem. A coordinate transformation was applied, ${ }^{14}$ to a reference $Y$ coordinate as follows

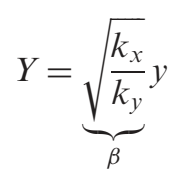

where $\beta=\sqrt{k_{x} / k_{y}}$ is the permeability anisotropy ratio. In this newly defined $\left(\boldsymbol{e}_{x}, \boldsymbol{e}_{Y}\right)$ reference frame, the constitutive equation (8) becomes

$\frac{\partial}{\partial x}\left(\frac{k_{x}}{\mu} \frac{\partial P}{\partial x}\right)+\frac{\partial}{\partial Y}\left(\frac{k_{x}}{\mu} \frac{\partial P}{\partial Y}\right)=\nabla_{\left(\boldsymbol{e}_{x}, \boldsymbol{e}_{Y}\right)} \cdot\left(\frac{k_{x}}{\mu} \nabla_{\left(\boldsymbol{e}_{x}, \boldsymbol{e}_{Y}\right)} P\right)=0$

where $\nabla_{\left(\boldsymbol{e}_{x}, \boldsymbol{e}_{Y}\right)}$ is the spatial derivation operator in the reference frame.

As a result of this transformation, the constitutive equation is isotropic in the reference frame.
Nonetheless the injection hole is no longer circular, but now described as an ellipse. The initial transition phase, as defined by Wang et al., ${ }^{34}$ in the vicinity of the injection hole, is very short and the isobars quickly become circular. Therefore, to model only the circular isobar phase, the injection hole was modified such that it was a circle in the reference frame. The impact of this transformation is negligible because the radius $r_{0}=$ $6 \mathrm{~mm}$ of the injection hole is much smaller than the overall preform dimension of $180 \mathrm{~mm}$. Furthermore, the anisotropy factor $\beta$ is close to 1 as reported in Table 2. To keep an equal perimeter, the radius of the injection hole is modified to $\widetilde{r_{0}}=\sqrt{\beta} \times r_{0}$. In the physical frame, the injection hole is modified to an ellipse of major axis $\sqrt{\beta} \times r_{0}$ and minor axis $r_{0} / \sqrt{\beta}$. The problem is now fully isotropic and invariant to rotations about the central point.

\section{Pressure field}

In the reference frame, at a given time, the relative pressure $P$ is a function of radial distance $r$ only. Relative injection pressure $P_{i n j}$ is imposed on radius $r=\widetilde{r_{0}}$ (injection hole) and atmospheric pressure 0 imposed at the flow front $r=l$. The boundary conditions thus write

$$
\left\{\begin{array}{c}
P\left(r=\tilde{r}_{0}\right)=P_{i n j} \\
P(r=l)=0
\end{array}\right.
$$

The constitutive equation (17) in polar coordinate writes

$$
\frac{1}{r} \frac{\partial}{\partial r}\left(r k \frac{\partial P}{\partial r}\right)=0
$$

which can be integrated easily using the boundary conditions (18) and give the pressure field as a function of the radial coordinate and the flow front position $l$ as

$$
P=P_{i n j} \times \frac{\ln (r)-\ln (l)}{\ln \left(\widetilde{r_{0}}\right)-\ln (l)}
$$

\section{Flow front evolution}

The flow front velocity is the apparent fluid velocity at the flow front radius. Thus

$$
\frac{d l}{d t}=v(r)
$$

where the apparent velocity is given by the Darcy law (5) 


$$
v(r)=-\frac{k}{\mu} \frac{\partial P}{\partial r}
$$

The pressure derivative can be obtained from the analytical pressure field (20) at position $r=l$ and gives

$$
\frac{d l}{d t}=-\frac{k}{\mu} \frac{P_{i n j}}{\ln \left(\widetilde{r_{0}}\right)-\ln (l)} \times \frac{1}{l}
$$

This ordinary differential equation along with the initial flow front position $l(t=0)=\widetilde{r_{0}}$ describes the flow front evolution.

By defining the new variable $\lambda=l^{2} / \widetilde{r}_{0}^{2}$, the ordinary differential equation simplifies to the transcendental equation

$$
\frac{d \lambda}{d t}=\frac{1}{\gamma \ln (\lambda)}
$$

where

$$
\gamma=\frac{\mu{\widetilde{r_{0}}}^{2}}{4 k P_{i n j}}
$$

is the characteristic time. Using the initial condition $\lambda(t=0)=1$, one gets the analytical formula for the flow front evolution as

$$
l(t)=\widetilde{r_{0}} \sqrt{\frac{t / \gamma}{W\left(\frac{t}{\gamma e}\right)}}
$$

where $e$ is the Euler number and $W$ is the Lambert function. ${ }^{35}$ 